\title{
Development of Optimal Water-Resources Management Strategies for Kaidu-Kongque Watershed under Multiple Uncertainties
}

\author{
Y. Zhou, ${ }^{1}$ Y. P. Li, ${ }^{1}$ G. H. Huang, ${ }^{1}$ and Y. Huang ${ }^{2}$ \\ ${ }^{1}$ MOE Key Laboratory of Regional Energy and Environmental Systems Optimization, \\ Resources and Environmental Research Academy, North China Electric Power University, Beijing 102206, China \\ ${ }^{2}$ State Key Laboratory of Desert and Oasis Ecology, Xinjiang Institute of Ecology and Geography, \\ Chinese Academy of Sciences, Urumqi 830011, China
}

Correspondence should be addressed to Y. P. Li; yongping.li33@gmail.com

Received 15 February 2013; Accepted 12 April 2013

Academic Editor: Xiaosheng Qin

Copyright (C) 2013 Y. Zhou et al. This is an open access article distributed under the Creative Commons Attribution License, which permits unrestricted use, distribution, and reproduction in any medium, provided the original work is properly cited.

In this study, an interval-stochastic fractile optimization (ISFO) model is advanced for developing optimal water-resources management strategies under multiple uncertainties. The ISFO model can not only handle uncertainties presented in terms of probability distributions and intervals with possibility distribution boundary, but also quantify subjective information (i.e., expected system benefit preference and risk-averse attitude) from different decision makers. The ISFO model is then applied to a real case of water-resources systems planning in Kaidu-kongque watershed, China, and a number of scenarios with different ecological waterallocation policies under varied p-necessity fractiles are analyzed. Results indicate that different policies for ecological water allocation can lead to varied water supplies, economic penalties, and system benefits. The solutions obtained can help decision makers identify optimized water-allocation alternatives, alleviate the water supply-demand conflict, and achieve socioeconomic and ecological sustainability, particularly when limited water resources are available for multiple competing users.

\section{Introduction}

The combined pressures from increasing water demands and limited water supplies have forced planners to contemplate comprehensive and efficient schemes for water resources management, especially in arid and semiarid regions $[1,2]$. Nowadays, continuing economic growth and rapid population increase are likely to aggravate water-shortage problems that may lead to controversial and conflict-laden waterallocation issues among multiple competing interests (e.g., municipal, industrial, stockbreeding, forestry, ecological, and agricultural). Moreover, more and more arid inland watersheds suffer from ecological degradation and vegetation losses as a result of limited water resources, severe weather conditions, poor management practices, and failed policy instructions [3-5]. Consequently, the conflicts between environmental protection and economic development in arid and semiarid regions have been pressing challenges for decision makers. Efficient and equitable water-resources management is required for regional socioeconomic and environmental sustainability.

Previously, a large number of modeling approaches based on stochastic mathematical programming (SMP) were advanced for allocating and managing water resources in more effective and sustainable ways [6-14]. For example, Watkins Jr et al. [15] proposed a scenario-based multistage stochastic programming model for planning water supplies from highland lakes. By explicitly considering a number of inflow scenarios, the stochastic model could help determine a contract for water delivery in the coming year. Harrison [16] advanced a chance-constrained linear programming model to allocate available land and water resources optimally on seasonal basis so as to maximize the net annual return from the study area, where net irrigation water requirements of crops were considered as stochastic variable. Li et al. [17] proposed a multistage fuzzy-stochastic programming model for supporting water resources management under uncertainty, which could tackle uncertainties expressed as 
fuzzy sets and probability distributions. Xu et al. [18] put forward an interval-parameter stochastic chance-constrained programming model for supporting multilayer urban watersupply system management under multiple uncertainties. Housh et al. [19] developed a limited multistage stochastic programming model for managing water-supply systems, where the number of decision variables at each stage remains constant, and thus the total number of decision variables increased only linearly as the number of scenarios and stages grew. Generally, SMP (i.e., multistage stochastic programming (MSP)) approach allows recourse actions to be undertaken in each stage based on updated information for realized system targets that take away the uncertainties, which could effectively reflect dynamic variations of multiple system components or conditions [20,21]. Nevertheless, the main limitation of conventional SMP approach is that the probability distributions of uncertain parameters may not be available due to the inadequate information; even if these distributions were known, reflection of them in large-scale stochastic models could be extremely challenging [22].

Consequently, an attractive technique named fractile optimization (FO) approach can solve the above limitations, which is an effective solution algorithm of fuzzy possibilistic programming (FPP) and could enable the flexible estimation of results with high satisfaction degree [23-26]. FO approach represents the possible or necessity degree of event occurrence for imprecise data described by fuzzy possibility distributions which can be obtained rather easily from decision makers' perception owing to the ordinality of possibility [27]. Inuiguchi and Ramík [23] proposed the concept of fractile which ranges between 0 and 1 and expresses the decision makers' preferences (i.e., risk-averse attitudes) and necessity or possibility degree towards the objective function. Katagiri et al. [28] advanced an interactive fuzzy satisficing method for solving a fuzzy random multiobjective linear programming problem, while the developed method is based on fractile optimization model with possibility and necessity measures, and the uncertain objective function could be transformed into a deterministic one with high satisfaction degree. Zhang et al. [29] developed a robust fractile optimization model for water-quality management, where the fractile optimization approach could be beneficial for acquiring maximized agricultural income and decreasing the system violation risk. Although the FO approach could be effective for handling the ambiguous parameters in the objective function, it has difficulties in dealing with uncertainties presented as interval numbers and/or probability distributions in a nonfuzzy decision space [23].

Actually, in real-world water-resources management problems, uncertainties could influence desired decision makings and they may exist in the system components, their interactions and their economic implications, such as water availability, economic parameters, water allocations, subjective judgments, sustainability requirements, and policy regulations. Some uncertainties can be quantified as single uncertainties (i.e., discrete intervals, probabilities) while the others may be expressed as dual uncertainties (i.e., intervals with possibility distribution boundary). For instance, fluctuating stream inflows may be presented as random variables with known probabilities; also, the water-allocation targets are difficult to be promised as deterministic values when available water resources are uncertain, and they may be obtained as interval numbers. Meanwhile, water would be allocated to multiple users over a multiperiod planning horizon, while the uncertainties can result in dynamic waterallocation schemes and varied water shortages. Moreover, owing to the intrinsic fluctuations of cash flow, economic parameters are often estimated by experts with interval values, and, at the same time, their lower and upper bounds of these intervals may be provided as subjective judgments from a number of decision makers (i.e., expressed as possibility distributions). The conventional FO approach may become infeasible in handling such dual uncertainties presented in terms of intervals with possibility distribution boundary. One potential approach is to integrate interval-parameter programming (IPP), MSP, and FO approach to effectively address such single and dual uncertainties. IPP approach is conducive to tackling the uncertainties expressed as crisp interval values in objective function and constraints without probability distributions and membership functions [30, 31].

Therefore, the objective of this study aims to develop an interval-stochastic fractile optimization (ISFO) model for optimal water-resources management strategies under multiple uncertainties. The ISFO model can not only handle uncertainties presented in terms of probability distributions and intervals with possibility distribution boundary, but also quantify subjective information (i.e., expected system benefit preference and risk-averse attitude) from different decision makers. The ISFO model will be applied to a real case of water-resources systems planning in the Kaidu-kongque watershed, a region threatened by serious water shortages and extremely vulnerable ecological system, while the continued vegetation degradation and human-induced soil erosion lead to increasingly intensified conflict between ecological preservation and economic development in water resources utilization. The obtained results with various $p$-necessity fractiles will be beneficial for identifying optimized water-allocation alternatives, alleviating the water supply-demand conflict, and achieving socioeconomic and ecological sustainability, particularly when limited water resources are available for multiple competing users.

The paper will be organized as follows: Section 2 describes the development process of ISFO model, where multiple uncertainties are considered; Section 3 presents the application in Kaidu-kongque watershed through the developed model; Section 4 provides result analysis and discussion, where a number of solutions associated with robust reflection of multiple uncertainties in relation to water-resources allocation, water-management policy, benefit analysis and riskaverse level are analyzed; Section 5 draws some conclusions.

\section{Model Development}

The study problem under consideration is how to allocate water from upstream of river to multiple users to maximize expected system net benefit and achieve environmental sustainability over a multiperiod planning horizon. In case of insufficient water availability, competition for water exists 
among different users. When the promised water is not delivered and the available water cannot meet the demands, the users will have to face a dilemma of either obtaining water from higher-priced schemes or curb the development plans, leading to incremental expenses or diminished benefits [32]. For instance, industries may have to decrease production levels, and farmlands may not be irrigated as planned. It is thus essential for allocating available water in more sustainable ways to reduce the related punishments. Being presented as random variables, the stream flows of the river may vary in various flow levels during different periods, which result in the dynamic water-allocation schemes. The associated decisions must be made at discrete points in discrete probability levels. In addition, owing to the intrinsic fluctuations of influence factors (e.g., cash flow and production price) and incomplete and imprecise datum, many coefficients and variables (i.e., water-allocation target, water availability, and economic parameter) are difficult for obtaining deterministic values and are thus expressed by interval numbers. Therefore, an interval-stochastic programming model can be formulated for study problem associated with multiple uncertainties:

$$
\begin{aligned}
\operatorname{Max} f^{ \pm}= & \sum_{i=1}^{I} \sum_{t=1}^{T} S Y_{i t}^{ \pm} \cdot F T_{i t}^{ \pm} \\
& -\sum_{i=1}^{I} \sum_{t=1}^{T} \sum_{k=1}^{K_{t}} p_{t k} E P_{i t}^{ \pm} \cdot W D_{i t k}^{ \pm}
\end{aligned}
$$

subject to

$$
\begin{aligned}
& \sum_{i=1}^{I}\left(F T_{i t}^{ \pm}-W D_{i t k}^{ \pm}\right) \leq W A_{t h}^{ \pm}+E W_{(t-1) k}^{ \pm}, \\
& \forall h, k=1,2, \ldots, K_{t} ; t=1,2, \ldots, T, \\
& E W_{(t-1) k}^{ \pm}=W A_{(t-1) h}^{ \pm}-\sum_{i=1}^{I}\left(F T_{i(t-1)}^{ \pm}-W D_{i(t-1) k}^{ \pm}\right) \\
& \quad+E W_{(t-2) k}^{ \pm}, \quad \forall h, k=1,2, \ldots, K_{t-1}, \\
& F T_{i t \max } \geq F T_{i t}^{ \pm} \geq W D_{i t k}^{ \pm}, \quad \forall i, t, k=1,2, \ldots, K_{t}, \\
& F T_{i t}^{ \pm}-W D_{i t k}^{ \pm} \geq M W R_{i t}, \quad \forall t, k=1,2, \ldots, K_{t}, \\
& F T_{i t}^{ \pm}, W D_{i t k}^{ \pm} \geq 0, \quad \forall i, t, \quad k=1,2, \ldots, K_{t},
\end{aligned}
$$

where $f^{ \pm}$is the net system benefit over the planning horizon $(\$) ; i$ is the water user, $i=1,2, \ldots, I ; t$ is the planning time period, $t=1,2, \ldots, T$; $h$ is the available flow level $(h=1,2$, $\ldots, H)$, with $h=1$ for the lowest flow and $h=H$ for the highest flow; $K_{t}$ is the number of scenarios in period $t$ and should be related to $H_{t}$, and the total number of scenarios is $K=\sum_{t=1}^{T} K_{t}=1 ; W A_{t h}^{ \pm}$is the water availability with probability level of $p_{t k}$ for $K_{t}$ scenario at each time stage $(t)$ $\left(10^{6} \mathrm{~m}^{3}\right) ; S Y_{i t}^{ \pm}$is the net benefits to user $i$ per unit of water allocated during period $t\left(\$ / 10^{3} \mathrm{~m}^{3}\right) ; E P_{i t}^{ \pm}$is the reduction of net benefit to user $i$ per unit of water not delivered during period $t\left(C_{i t}>B_{i t}\right)\left(\$ / 10^{3} \mathrm{~m}^{3}\right) ; p_{t k}$ is the probability of occurrence for scenario $k$ in period $t$, with $p_{t h}>0$ and $\sum_{K=1}^{K_{i}} p_{t k}=1 ; E W_{(t-1) k}^{ \pm}$is the surplus water in the reservoir when water is delivered in period $t-1$ under scenario $k\left(10^{6} \mathrm{~m}^{3}\right) ; F T_{i t \max }$ is the maximum allowable allocation amount for user $i$ in period $t\left(10^{6} \mathrm{~m}^{3}\right) ; M W R_{i t}$ is the minimum amount that should be allocated to user $i$ in period $t\left(10^{6} \mathrm{~m}^{3}\right) ; F T_{i t}^{ \pm}$is the fixed water allocation target that is, promised to user $i$ during period $t\left(10^{6} \mathrm{~m}^{3}\right) ; W D_{i t k}^{ \pm}$is the amount by which the water-allocation targets are not met when the seasonal flows are $q_{t h}\left(10^{6} \mathrm{~m}^{3}\right)$.

Obviously, model ((1a), (1b), (1c), (1d), (1e), (1f)) can be effective for handling uncertainties presented as crisp interval values in objective function and constraints and random variables in the right-hand sides with known probability distributions. However, modeling parameters may be provided as subjective judgments from a number of stakeholders and decision makers (i.e., expressed as possibility distributions), which makes interval-stochastic programming model become infeasible [33]. Therefore, fractile optimization (FO) approach, based on the fuzzy possibilistic programming (FPP), can effectively solve uncertainties presented as possibility distributions, while its necessity is described as the treatment of an objective function [23-26, 34]. When uncertainties are expressed as possibility distributions in the ambiguous coefficients of objective function, a general FPP model can be formulated as follows [35-37]:

$$
\operatorname{Max} f=\underset{\sim}{C X}
$$

subject to

$$
\begin{gathered}
A X \leq B \\
X \geq 0
\end{gathered}
$$

where coefficient $\underline{C}$ represents the fuzzy possibilistic variables restricted by fuzzy triangular numbers with possibility distribution. Generally, possibility distribution can be regarded as fuzzy membership function, and possibility degree can be considered as the membership value [38]. In virtue of the computational efficiency and simplicity in data acquisition, a symmetric triangular fuzzy number $\underset{C}{ }$ is considered, which can be determined by a center $c^{c}$ and a spread $w$, and can be described as $\underline{C}=\left(c^{c}, w\right)[34,39]$. Accordingly, the linear objective function of the model ((2a), (2b), (2c)) with ambiguous parameters (i.e., Equation $(2 \mathrm{a})$ ) can be transformed as follows:

$$
\operatorname{Max} f=\left(c^{c} X, w|X|\right)
$$

In the possibility theory, necessity measure is defined as follows $[23,34,35]$ :

$$
N_{C}(B)=\inf _{r} \max \left(1-\mu_{C}(r), \mu_{B}(r)\right),
$$

where $\mu_{B}$ is the membership function of the fuzzy set $B$; $N_{C}(B)$ denotes the certainty (or necessity) degree of the event 
that fuzzy possibilistic variable $\underline{C}$ restricted by the possibility distribution $\mu_{C}$ is in the fuzzy set $B$. Let $B=(-\infty, u]$ or $[u,+\infty)$, which is indicated as a crisp set of real numbers which is not greater (or not smaller) than $u$. Then, we obtain the following indices by necessity measure defined by (5) and (6) $[23,34]$ :

$$
\begin{aligned}
& \operatorname{Nes}(\underset{C}{C} u)=N_{C}((-\infty, u])=1-\sup \left\{\mu_{C}(r) \mid r>u\right\}, \\
& \operatorname{Nes}(\underset{\sim}{C} \geq u)=N_{C}([u,+\infty))=1-\sup \left\{\mu_{C}(r) \mid r<u\right\} .
\end{aligned}
$$

Based on the definition of necessity measure mentioned above, the $p$-necessity fractile is defined as follows [33]:

$$
\operatorname{Nes}(\underset{\sim}{f \geq u}) \geq p_{\text {nes }}
$$

where $u$ denotes the $p$-fractile value; $\underset{\sim}{f}$ means the fuzzy possibilistic variable (i.e., model (2a)) restricted by fuzzy numbers; the value of necessity measure (i.e., Nes (.)) belongs to the interval $[0,1]$. Given the appropriate level $p_{\text {nes }}[0,1]$, the problem is transformed to maximize the $p$-fractile value under the condition that a necessity measure of the event that the objective function value is not lesser than $p$-fractile value $u$ is greater than or equal to $p_{\text {nes }}$. The diverse $p_{\text {nes }}$ levels express the decision makers' preferences toward the objective function value, which implies the certainty (necessity) degree of the objective function (also named $p$-necessity level). In real-world applications, the decision makers prefer that the objective function should be satisfied under a high certainty degree (high $p$-necessity level). Given the $p$-necessity level, the problem (i.e., model (2a), (2b), (2c)) can be transformed into the following linear necessity fractile optimization model with deterministic objective:

$$
\operatorname{Max} u
$$

subject to

$$
\text { Nes }\left\{\left(c^{c} X, w|X|\right) \geq u\right\} \geq p_{\text {nes }} .
$$

Based on the fractile optimization model (as illustrated in Figure 1), the problem is to maximize the $p$-necessity fractile of a possibilistic variable $\underset{\sim}{f}$, and model (5) corresponds to

$$
\operatorname{Max}\left(c^{c} X-p_{\text {nes }} w|X|\right) .
$$

In practical water resources systems planning, interval parameters (i.e., economic data) may fluctuate with their bounds being available as subjective preferences (i.e., expected system benefit standpoint and risk-averse attitude), which may be provided by possibility distributions. The concept of intervals with possibility distribution boundary is proposed in this study to represent the dual uncertainties in the system components. Consequently, $\mathrm{FO}$ approach is introduced into the above interval-stochastic programming framework to

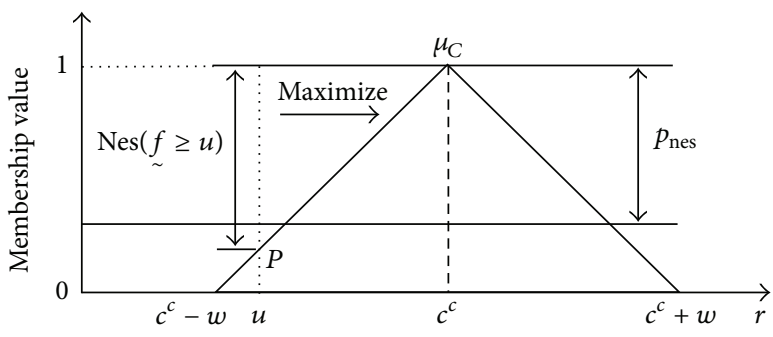

FIgURE 1: Fractile optimization model.

handle such dual uncertainties in the objective function. This leads to an interval-stochastic fractile optimization (ISFO) model as follows:

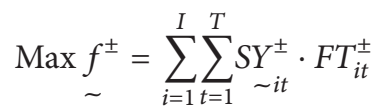

$$
\begin{aligned}
& -\sum_{i=1}^{I} \sum_{t=1}^{T} \sum_{k=1}^{K_{t}} p_{t k} E P_{\sim i t}^{ \pm} \cdot W D_{i t k}^{ \pm}
\end{aligned}
$$

subject to

$$
\begin{aligned}
& \sum_{i=1}^{I}\left(F T_{i t}^{ \pm}-W D_{i t k}^{ \pm}\right) \leq W A_{t h}^{ \pm}+E W_{(t-1) k}^{ \pm}, \\
& \forall h, k=1,2, \ldots, K_{t}, \quad t=1,2, \ldots, T, \\
& E W_{(t-1) k}^{ \pm}=W A_{(t-1) h}^{ \pm}-\sum_{i=1}^{I}\left(F T_{i(t-1)}^{ \pm}-W D_{i(t-1) k}^{ \pm}\right) \\
& +E W_{(t-2) k}^{ \pm}, \quad \forall h, k=1,2, \ldots, K_{t-1},
\end{aligned}
$$

$$
F T_{i t \max } \geq F T_{i t}^{ \pm} \geq W D_{i t k}^{ \pm}, \quad \forall i, t, k=1,2, \ldots, K_{t},
$$

$$
F T_{i t}^{ \pm}-W D_{i t k}^{ \pm} \geq M W R_{i t}, \quad \forall t, k=1,2, \ldots, K_{t},
$$

$$
F T_{i t}^{ \pm}, W D_{i t k}^{ \pm} \geq 0, \quad \forall i, t, k=1,2, \ldots, K_{t} .
$$

For model (10a), (10b), (10c), (10d), (10e), (10f), in order to identify an optimized set of target values (i.e., $F T_{t}^{ \pm}$), $z_{t}$ are introduced to be decision variables into models (10a), (10b), (10c), (10d), (10e), and (10f). Let $F T_{t}^{ \pm}=F T_{t}^{-}+\Delta F T_{t} \cdot z_{t}$, where $\Delta F T_{t}=F T_{t}^{+}-F T_{t}^{-}$and $z_{t} \in[0,1]$. Thus, when $F T_{t}^{ \pm}$ reach their upper bounds (i.e., when $z_{t}=1$ ), a relatively high objective function value will be gained in the case of satisfactory water demand; an associated high penalty may have to be paid when the promised water is not delivered. On the contrary, when $F T_{t}^{ \pm}$approach their lower bounds (i.e., when $z_{t}=0$ ), a lower objective function value and related lower risk will be obtained. Accordingly, through introducing decision variables $z_{t}$, the ISFO model can be transformed into two deterministic submodels. 
Submodels (11a), (11b), (11c), (11d), (11e), and (11f):

$$
\begin{aligned}
\operatorname{Max} f^{+}= & \sum_{i=1}^{I} \sum_{t=1}^{T} S Y_{i t}^{c+}\left(F T_{i t}^{-}+\Delta F T_{i t} \cdot z_{t}\right) \\
& -\sum_{i=1}^{I} \sum_{t=1}^{T} p_{\mathrm{nes}} w_{S Y}\left|\left(F T_{i t}^{-}+\Delta F T_{i t} \cdot z_{t}\right)\right| \\
& -\sum_{i=1}^{I} \sum_{t=1}^{T} \sum_{k=1}^{K_{t}} p_{t k} E P_{i t}^{c-} W D_{i t k}^{-} \\
& +\sum_{i=1}^{I} \sum_{t=1}^{T} \sum_{k=1}^{K_{t}} p_{t k} p_{\mathrm{nes}} w_{E P}\left|W D_{i t k}^{-}\right|
\end{aligned}
$$

subject to

$$
\begin{gathered}
\sum_{i=1}^{I}\left[\left(F T_{i t}^{-}+\Delta F T_{i t} \cdot z_{t}\right)-W D_{i t k}^{-}\right] \leq W A_{t h}^{+}+E W_{(t-1) k}^{+} \\
\forall h, k=1,2, \ldots, K_{t}, t=1,2, \ldots, T \\
E W_{(t-1) k}^{+}=W A_{(t-1) h}^{+}-\sum_{i=1}^{I}\left[\left(F T_{i t}^{-}+\Delta F T_{i t} \cdot z_{t}\right)-W D_{i t k}^{-}\right] \\
+E W_{(t-2) k}^{+}, \quad \forall h, k=1,2, \ldots, K_{t-1},
\end{gathered}
$$

$F T_{i t \text { max }} \geq F T_{i t}^{-}+\Delta F T_{i t} \cdot z_{t} \geq W D_{i t k}^{-}, \quad \forall i, t, k=1,2, \ldots, K_{t}$,

$$
\begin{array}{r}
\left(F T_{i t}^{-}+\Delta F T_{i t} \cdot z_{t}\right)-W D_{i t k}^{-} \geq M W R_{i t}, \\
\forall t, k=1,2, \ldots, K_{t},
\end{array}
$$

$F T_{i t}^{-}+\Delta F T_{i t} \cdot z_{t} \geq 0, \quad W D_{i t k}^{-} \geq 0, \quad \forall i, t, k=1,2, \ldots, K_{t}$.

Submodels (12a), (12b), (12c), (12d), (12e), and (12f):

$$
\begin{aligned}
\operatorname{Max} f^{-}= & \sum_{i=1}^{I} \sum_{t=1}^{T} S Y_{i t}^{c-}\left(F T_{i t}^{-}+\Delta F T_{i t} \cdot z_{t \text { opt }}\right) \\
& -\sum_{i=1}^{I} \sum_{t=1}^{T} p_{\text {nes }} w_{S Y}\left|\left(F T_{i t}^{-}+\Delta F T_{i t} \cdot z_{t \mathrm{opt}}\right)\right| \\
& -\sum_{i=1}^{I} \sum_{t=1}^{T} \sum_{k=1}^{K_{t}} p_{t k} E P_{i t}^{c+} W D_{i t k}^{+} \\
& +\sum_{i=1}^{I} \sum_{t=1}^{T} \sum_{k=1}^{K_{t}} p_{t k} p_{\mathrm{nes}} w_{E P}\left|W D_{i t k}^{+}\right|
\end{aligned}
$$

subject to

$$
\begin{array}{r}
\sum_{i=1}^{I}\left[\left(F T_{i t}^{-}+\Delta F T_{i t} \cdot z_{t \mathrm{opt}}\right)-W D_{i t k}^{+}\right] \leq W A_{t h}^{-}+E W_{(t-1) k}^{-}, \\
\forall h, k=1,2, \ldots, K_{t}, t=1,2, \ldots, T,
\end{array}
$$

$$
\begin{aligned}
& E W_{(t-1) k}^{-}=W A_{(t-1) h}^{-}-\sum_{i=1}^{I}\left[\left(F T_{i t}^{-}+\Delta F T_{i t} \cdot z_{t \text { opt }}\right)-W D_{i t k}^{+}\right] \\
& +E W_{(t-2) k}^{-}, \quad \forall h, k=1,2, \ldots, K_{t-1}, \\
& F T_{i t \max } \geq F T_{i t}^{-}+\Delta F T_{i t} \cdot z_{t \text { opt }} \geq W D_{i t k}^{+} \text {, } \\
& \forall i, t, k=1,2, \ldots, K_{t} \text {, } \\
& \left(F T_{i t}^{-}+\Delta F T_{i t} \cdot z_{t \mathrm{opt}}\right)-W D_{i t k}^{+} \geq M W R_{i t}, \\
& \forall t, k=1,2, \ldots, K_{t}, \\
& F T_{i t}^{-}+\Delta F T_{i t} \cdot z_{\text {topt }} \geq 0, \\
& W D_{i t k}^{+} \geq 0, \quad \forall i, t, k=1,2, \ldots, K_{t} \text {. }
\end{aligned}
$$

By solving above two submodels, the final solutions for ISFO model $f_{\text {opt }}^{ \pm}=\left[f_{\text {opt }}^{-}, f_{\text {opt }}^{+}\right], F T_{i t \text { opt }}^{ \pm}=F T_{i t}^{-}+\Delta F T_{i t} \cdot z_{t \text { opt }}$ and $W D_{i t k \text { opt }}^{ \pm}=\left[W D_{i t k \text { opt }}^{-}, W D_{i t k \text { opt }}^{+}\right]$can be acquired. The actual water allocation would be the difference between the promised target values (i.e., $F T_{i t \text { opt }}^{ \pm}$) and the shortage values (i.e., $W D_{i t k \mathrm{opt}}^{ \pm}$).

\section{Case Study}

Kaidu-Kongque watershed (within $\mathrm{E} 85^{\circ} 20^{\prime}-87^{\circ} 30^{\prime}, \mathrm{N} 41^{\circ} 10^{\prime}-$ $42^{\circ} 30^{\prime}$ ), one of mainstreams of the Tarim River, is situated in the Bayangol Mongol autonomous prefecture in Xinjiang Uygur autonomous region of northwest China. As the upper reach of the watershed, Kaidu River originates from Saaer mountain of the middle Tianshan Mountains, passes through Yourdusi basin, enters Yanqi basin, flows into Bosten Lake, then is pumped into Kongque River at southwest of the lake, crosses Kezile mountain of the south Tianshan Mountains, and finally reaches Tarim basin. The watershed contains one city (i.e., Ku'erle city), five countries (i.e., Yanqi, Hejing, Heshuo, Bohu, and Weili), and ten production and construction corps, covering an area of approximately $31.40 \times 10^{3} \mathrm{~km}^{2}$. A temperate continental arid climate characterizes the watershed. The annual average precipitation ranges from $47.3 \mathrm{~mm}$ to $75.0 \mathrm{~mm}$ and mainly concentrates in May and October, indicating uneven distribution of precipitation through the year. The annual average pan evaporation ranges from $1887 \mathrm{~mm}$ to $2777 \mathrm{~mm}$. The watershed is mainly recharged by rainfall and alpine glacier-snow melt water, while the gross amount of water resources of the watershed is approximately $4.41 \times 10^{9} \mathrm{~m}^{3}$. The water from upstream of Kaidu-kongque River is allocated to six users, including municipal, industrial, stockbreeding, forestry, agricultural, and ecological sectors (as shown in Figure 2). At the end of 2008, the total population of the watershed was 1.03 million, and the gross industrial output value reached RMB $¥ 8.37$ billion. Besides, the largest alpine meadow of China, named Bayanbulak Grassland, is located in the watershed, with a total area of $23.00 \times 10^{3} \mathrm{~km}^{2}$, which is beneficial for stockbreeding. Moreover, owing to suitable environmental conditions in the lower beach of Kaidu River, 


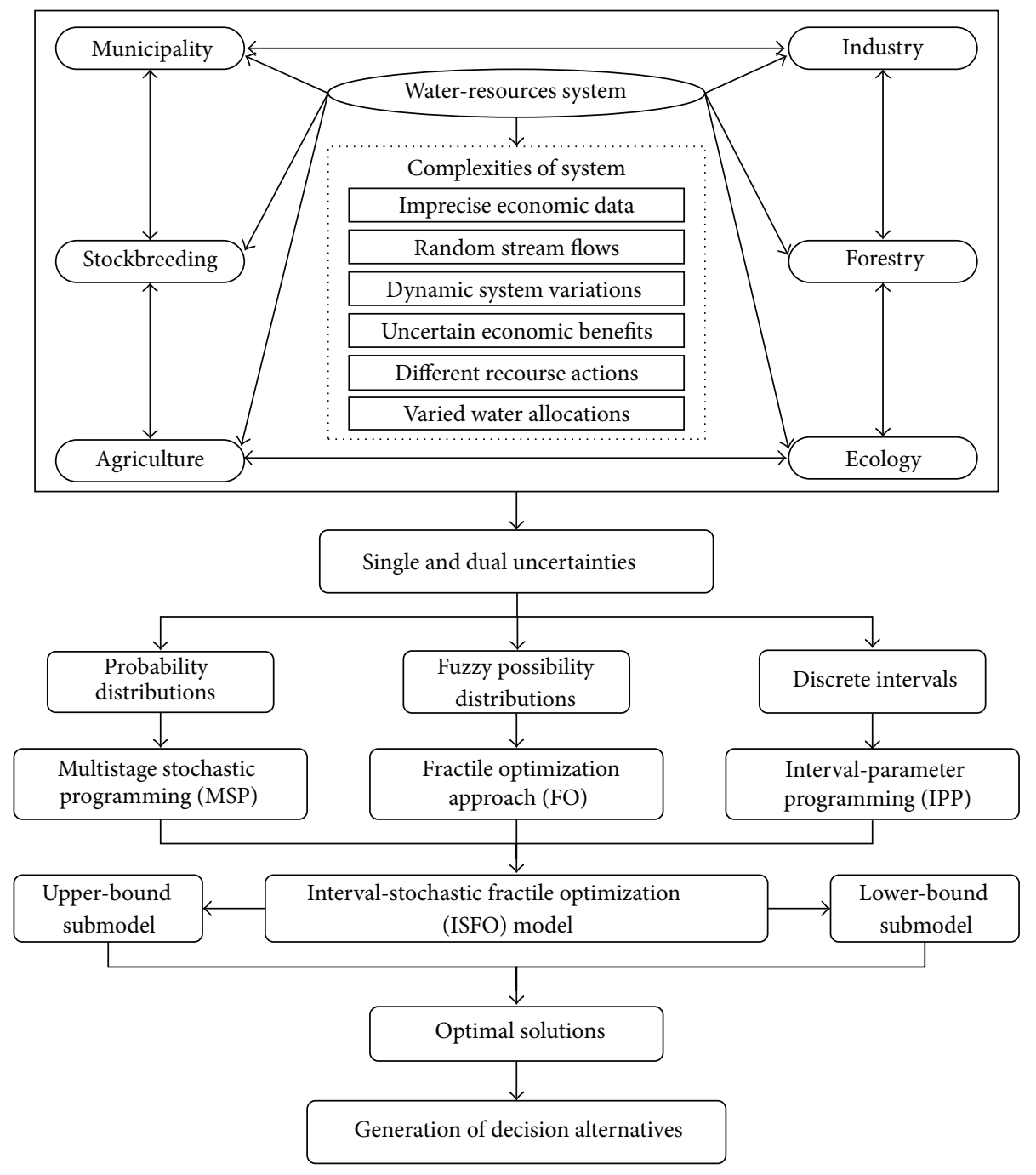

FIGURE 2: Diagram of planning water-resources system.

the Kaidu-kongque watershed has been a significant region for agricultural production, processing and exporting in Xinjiang province. At the same time, the ecosystems in this region are extremely vulnerable to disturbances and habitat degradation owing to severe water resource shortage [40]. Being in the middle reach of the Tarim River basin, the watershed is the supplement for ecosystem recovering of the lower reach of the Tarim River.

In recent years, with incremental population growth, rapid economic development and accelerating industrialization and urbanization processes, water demand of the watershed has been experienced a continuous and significant increment. Nevertheless, past practice experience and sustainable development strategies in water resources management have raised increasing concerns in irrational utilization and over exploitation of water resources. Kaidu-kongque watershed confronts with serious water shortages, while agricultural sector contributes to the largest water consumer. Owing to the severe weather conditions with characteristics of scarce rainfall, high temperature and high evaporation in the region, over 95 percent of the arable land in the watershed needs irrigation [41]. The statistics suggest that agricultural irrigation area of the watershed ran up to $391.62 \times$ $10^{3} \mathrm{~m}^{2}$ in 2009 , while agricultural water consumption came up to $2.40 \times 10^{9} \mathrm{~m}^{3}$, accounting for the $96.31 \%$ of the total water consumption. Furthermore, the seasonal variation of stream flow leads to the water scarcity in spring and water surplus in summer which gives rise to water utilization conflicts in local agricultural production. Consequently, under the circumstance of excessive agricultural water consumption and unreasonable irrigation mode, the pressure on local water resources intensifies, resulting in tensions, conflicts among water users, and excessive pressure on ecological environment. Specifically, natural variability and intensive human activities (i.e., excessive water withdrawal and consumption in upper and middle reaches of the river) have led to a large decrease in streamflow, dried-up riverbed, and withered natural vegetations (i.e., populus euphratica and meadow) in the lower beaches of river, which further aggravate the ecological degradation. Such irrational water 
utilization and over-exploitation problems intensify the water supply-demand conflict, accelerate the ecological environment destruction, threaten the sustainable development, and even have far-reaching impacts on watershed environment and human beings' lives. Unfortunately, in this region, there is a lack of effective plan for facilitating efficient, equitable and sustainable water resources management. Previous waterresources management in the watershed is mostly based on statistical analyses of historical data. Therefore, in order to recovery the ecological system in the lower reaches of the watershed, it is essential to develop an effective modeling technique for supporting water resources management under such conflicts.

As shown in Figure 2, the water resources system of the study area could be considered as an interrelated network connected by various system components. It is indicated that all kinds of complexities may exist in the study system, including imprecise economic data, random stream flows, dynamic system variables, uncertain economic benefits, various recourse actions, and varied water allocations. These components and their interactions must be systematically estimated in planning an integrated water resources management model within multiperiod. Specifically, due to spatial and temporal variations of precipitation, evapotranspiration and snow melting in the study system, the stream flow of Kaidu-kongque River presents a remarkable pattern of seasonal variation. Summer stream flow contributes to the greatest proportion, amounting to $45.0 \%$, followed by spring stream flow $(23.2 \%)$, fall stream flow $(21.1 \%)$, and winter stream flow (10.7\%). Accordingly, the fluctuating stream flows are expressed as with known probabilities, which may lead to varied water-allocation plans and various recourse actions. Moreover, several economic implications associated with economic fluctuations and subjective judgments could bring about uncertain system benefits and affect waterallocation decision makings. Imprecise economic data are highly uncertain and represented as intervals with possibility distribution boundary; this results in dual uncertainties in the objective function. In order to generate a desired compromise between the required environmental objective and maximized system benefit, how to effectively handle such single and dual uncertainties is regarded as a primary concern. Comprehensive optimization models are effective methods for allocating and managing water resources in more efficient and environmentally benign ways.

In real-world water-resources management problems, uncertainties are often associated with various in terms of information quality [42]. The random characteristic of stream flow and ambiguity in benefits and penalties parameters are all possible sources of uncertainties. The stream flow is expressed as a random variable; its probability distribution is obtained by statistical analysis when the collection data are enough. However, due to inadequate information or subjective judgment of decision makers, possibilistic distributions of economic parameters could be estimated through a number of questionnaires. Generally, random parameters are expressed as probability density functions (PDFs), while possibility distribution can be regarded as fuzzy membership function [43].
In this study, the time horizon under consideration is 3 years, which is divided into three planning periods. Modeling parameters are estimated based on the statistical yearbook of Bayangol Mongol Autonomous Prefecture and Xinjiang Uygur Autonomous Region or derived from published reports and papers [41, 44]. For example, water availability is presented as random variable, which is obtained through the 46-year statistical analyses with simulation results of annual stream flow of the Kaidu-kongque River; based on the previous simulation results, after determining the probability distribution (i.e., gamma distribution) of the simulated stream flow, its discretization values with different probability levels can be acquired [41]. Table 1 presents discrete intervals of stream flows and associated probability levels during three planning periods. Table 2 provides the promised waterallocation targets as well as maximum allowable allocations from municipal, industrial, stockbreeding, forestry, agricultural, and ecological sectors, which is on the basis of previous studies [41]. Shortages in water supply will occur if available water is less than water demands, such that the promised targets cannot be delivered (i.e., water shortage $=$ promised target - available water). Under such circumstances, the actual water allocation will be the difference between the promised target and the probabilistic water shortage (i.e., actual water allocation $=$ promised target - water shortage) . Table 3 shows the relevant system benefits and penalties data, which are expressed by intervals with possibility distribution boundary. Specifically, the interval values of net benefit for each unit of water can be calculated by historical data through dividing the gross annual value of each water user by its annual water consumption, while the possibility distributions are estimated by the subjective judgments of 100 decision makers and stakeholders through 120 questionnaires. The economic penalties correspond to the acquisition of water from higher-priced alternatives and/or the negative consequences generated from the curbing of regional development plans when the promised water is not delivered [45]. Besides, a water-resources management policy based on minimum water requirement for ecological sector is considered through ISFO model, which complies with the policy on recovering the ecological system in the lower beaches of the river. Case 1 is based on the current water-resource allocation policies with $p$-necessity level of 0.90 , while $100 \times 10^{6} \mathrm{~m}^{3}$ of minimum water requirement for ecological sector is required to be satisfied (means the basic case); in Case 2, minimum water requirement for ecological sector is not considered in the ISFO model when $p$-necessity level $=0.90$.

\section{Results and Discussion}

4.1. Results under Basic Case. In this study, three stream flow levels (i.e., low, medium, and high) were considered in each period. Accordingly, a three-period (four-stage) scenario tree can be generated for each of water users, which resulted in 3 nodes (scenarios) in period 1 (stage 2 ), 9 nodes (scenarios) in period 2 (stage 3 ), and 27 nodes (scenarios) in period 3 (stage 4). The solutions obtained under basic case (e.g., Case 1) are presented in Figures 3-5. The results (i.e., objective function 
TABLE 1: Stream flows and associated probabilities.

\begin{tabular}{|c|c|c|c|c|c|c|}
\hline \multirow{2}{*}{ Flow level } & \multicolumn{2}{|r|}{$t=1$} & \multicolumn{2}{|r|}{$t=2$} & \multicolumn{2}{|r|}{$t=3$} \\
\hline & Probability & Stream flow $\left(10^{6} \mathrm{~m}^{3}\right)$ & Probability & Stream flow $\left(10^{6} \mathrm{~m}^{3}\right)$ & Probability & Stream flow $\left(10^{6} \mathrm{~m}^{3}\right)$ \\
\hline Low $(\mathrm{L})$ & 0.304 & {$[2459,2989]$} & 0.370 & {$[2399,3003]$} & 0.174 & {$[2602,2901]$} \\
\hline Medium (M) & 0.455 & {$[3002,3721]$} & 0.502 & {$[3062,3847]$} & 0.490 & {$[2917,3626]$} \\
\hline $\operatorname{High}(\mathrm{H})$ & 0.241 & {$[3752,5708]$} & 0.128 & {$[4068,5676]$} & 0.336 & {$[3700,5500]$} \\
\hline
\end{tabular}

TABLE 2: Water resource allocations for each sector (unit: $10^{6} \mathrm{~m}^{3}$ ).

\begin{tabular}{lccc}
\hline \multicolumn{3}{c}{ Time period } \\
& $t=1$ & $t=2$ & $t=3$ \\
\hline Municipality & {$[69,191]$} & {$[80,200]$} & {$[90,220]$} \\
Industry & {$[469,803]$} & {$[550,900]$} & {$[420,800]$} \\
Stockbreeding & {$[41,155]$} & {$[45,170]$} & {$[50,175]$} \\
Forestry & {$[407,527]$} & {$[460,588]$} & {$[480,600]$} \\
Agriculture & {$[2335,3113]$} & {$[2420,3200]$} & {$[2480,3350]$} \\
Ecology & {$[445,1051]$} & {$[500,1100]$} & {$[500,1100]$} \\
\hline \multicolumn{4}{c}{ Maximum allowable water allocation } \\
\hline Municipality & 200 & 200 \\
Industry & 900 & 900 & 200 \\
Stockbreeding & 200 & 200 & 900 \\
Forestry & 600 & 600 & 200 \\
Agriculture & 3500 & 3500 & 600 \\
Ecology & 1200 & 1200 & 3500 \\
\hline
\end{tabular}

value and decision variables) included the interval, probabilistic, and fuzzy possibilistic information, in which multiple uncertainties (i.e., probability distributions and intervals with possibility distribution boundary) can be facilitated to obtain multiple decision alternatives.

Figure 3 presents the optimized water-allocation targets for various users over the planning horizon under the basic case. The results indicate that the optimized water-allocation target for municipal sector would be $191 \times 10^{6}, 200 \times 10^{6}$ and $200 \times 10^{6} \mathrm{~m}^{3}$, which amount to their upper-bound targets in periods 1-2 and approaches the upper-bound target in period 3. Similarly, the optimized water-allocation targets for industrial, stockbreeding, and ecological sectors would also reach their upper-bounds. In comparison, the optimized water-allocation target for forestry sector would be in the range of its lower- and upper-bound targets for water resource allocations, amounting to $527 \times 10^{6}, 586 \times 10^{6}$, and $480 \times$ $10^{6} \mathrm{~m}^{3}$ during three periods; the optimized water-allocation target for agricultural sector would approach its lower-bound water-allocation target, reaching $2682 \times 10^{6}, 2420 \times 10^{6}$, and $2480 \times 10^{6} \mathrm{~m}^{3}$ over the planning horizon. This demonstrates that the planning allocation target for ecological sector would meet its water demand, while the planning allocation target for agricultural sector would not meet its essential demand, if a minimum amount of ecological water requirement was considered by the modeling formulation.
Shortages in water supply would occur if available stream flows could not satisfy the water-allocation targets, where the actual water-allocation plans of each user would be the difference between the optimized water-allocation target and the probabilistic water shortage. In case of insufficient water supply, the municipal sector should be the highest priority since it brings the highest benefits when water demand is satisfied, then to stockbreeding, industrial, agricultural, ecological, and forestry sectors. The solutions through ISFO model demonstrate that municipal, industrial and stockbreeding sectors would not be subject to any water deficit under all stream flows, while the water deficits would be occur for forestry, agricultural, and ecological sectors. This is attributed to the fact that the municipal, industrial and stockbreeding sectors bring relative high benefits when promised waters are delivered; meanwhile, they are subject to the high penalties the promised targets are not satisfied.

Figure 4 represents the water-allocation plans for agricultural sector over the planning horizon under the basic condition. By a three-period (four-stage) scenario tree, the solutions of water deficit could reflect the dynamic system variations. The maximum value of the deficit is associated with lower system benefit; contrarily, the minimum value of the deficit corresponds to higher system benefit. As shown in Figure 4, when stream flows are low in all of the three periods (i.e., under scenario LLL with a joint probability of $1.96 \%)$, the probabilistic water deficits for agricultural sector would be $[947,1477] \times 10^{6} \mathrm{~m}^{3}$ in period 1 (probability = $30.4 \%),[801,1405] \times 10^{6} \mathrm{~m}^{3}$ in period 2 (probability $\left.=11.2 \%\right)$, and $[868,1167] \times 10^{6} \mathrm{~m}^{3}$ in period 3 (probability $=1.96 \%$ ), respectively. Accordingly, the optimized water allocated to agricultural sector would be $[1205,1735] \times 10^{6} \mathrm{~m}^{3}$ in period $1,[1015,1619] \times 10^{6} \mathrm{~m}^{3}$ in period 2 , and $[1313,1612] \times 10^{6} \mathrm{~m}^{3}$ in period 3, respectively; the total water-allocation amount to this user would be $[3532,4965] \times 10^{6} \mathrm{~m}^{3}$ under this scenario. The solutions for water deficits and water-allocation plans under other scenarios could be similarly analyzed based on the solutions represented in Figure 4.

Besides, the solutions imply that varied seasonal flows result in different water allocations to various users. For instance, when stream flows are high in all of the three periods, the optimized water allocation to forestry sector would, respectively, be $[0,527] \times 10^{6} \mathrm{~m}^{3}$ in period 1 (probability $=$ $24.1 \%),[0,586] \times 10^{6} \mathrm{~m}^{3}$ in period 2 (probability $=3.08 \%$ ), and $[0,445] \times 10^{6} \mathrm{~m}^{3}$ in period 3 (probability $=1.04 \%$ ), while the optimized water allocation to ecological sector would be $[105,1051] \times 10^{6} \mathrm{~m}^{3}$ in period $1,[378,1100] \times 10^{6} \mathrm{~m}^{3}$ in period 2 , and $[114,1090] \times 10^{6} \mathrm{~m}^{3}$ in period 3 . When a 
TABLE 3: Net benefits and penalties (unit: $\$ / 10^{3} \mathrm{~m}^{3}$ ).

\begin{tabular}{|c|c|c|c|}
\hline & \multicolumn{3}{|c|}{ Time period } \\
\hline & $t=1$ & $t=2$ & $t=3$ \\
\hline \multicolumn{4}{|c|}{ Net benefit when water demand is satisfied } \\
\hline Municipality & $\begin{array}{c}{[(6443,7857,9271),(8200,10000,} \\
11800)]\end{array}$ & $\begin{array}{c}{[(5664,6907,8150),(8120,9903,} \\
11686)]\end{array}$ & $\begin{array}{c}{[(5920,7220,8520),(8202,10003,} \\
11804)]\end{array}$ \\
\hline Industry & $\begin{array}{c}{[(1693,1820,1947),(5965,6414,} \\
6863)]\end{array}$ & {$[(1767,1900,2033),(5859,6300,6741)]$} & {$[(1830,1968,2106),(6511,7001,7491)]$} \\
\hline Stockbreeding & $\begin{array}{c}{[(3510,3900,4290),(5837,6485,} \\
7134)]\end{array}$ & $\begin{array}{c}{[(3510,3900,4290),(5651,6279,} \\
6907)]\end{array}$ & $\begin{array}{c}{[(3447,3830,4213),(5400,6000,} \\
6600)]\end{array}$ \\
\hline Forestry & {$[(254,257,260),(366,371,376)]$} & {$[(284,288,292),(402,407,412)]$} & {$[(267,269,272),(387,392,397)]$} \\
\hline Agriculture & {$[(336,341,346),(448,455,462)]$} & {$[(362,367,373),(473,480,487)]$} & {$[(384,390,396),(547,555,563)]$} \\
\hline Ecology & {$[(155,157,159),(424,429,434)]$} & {$[(168,170,172),(514,520,526)]$} & {$[(189,191,193),(523,529,535)]$} \\
\hline \multicolumn{4}{|c|}{ Penalty when promised water is not delivered } \\
\hline Municipality & $\begin{array}{c}{[(12142,14285,16428),(15785,18571,} \\
21357)]\end{array}$ & $\begin{array}{c}{[(11121,13084,15047),(16148,18998,} \\
21848)]\end{array}$ & $\begin{array}{c}{[(12266,14430,16595),(16405,19300,} \\
22195)]\end{array}$ \\
\hline Industry & $\begin{array}{c}{[(3456,3600,3744),(10984,11442} \\
11900)]\end{array}$ & $\begin{array}{c}{[(3360,3500,3640),(11520,12000} \\
12480)]\end{array}$ & $\begin{array}{c}{[(3539,3687,3834),(12672,13200,} \\
13728)]\end{array}$ \\
\hline Stockbreeding & $\begin{array}{c}{[(7307,7857,8407),(11957,12857,} \\
13757)]\end{array}$ & $\begin{array}{c}{[(6975,7500,8025),(10560,11355,} \\
12150)]\end{array}$ & $\begin{array}{c}{[(7068,7600,8132),(11102,11938,} \\
12774)]\end{array}$ \\
\hline Forestry & {$[(511,515,519),(736,742,748)]$} & {$[(526,530,534),(794,800,806)]$} & {$[(516,520,524),(774,780,786)]$} \\
\hline Agriculture & {$[(636,642,648),(990,1000,1010)]$} & {$[(713,720,727),(1089,1100,1111)]$} & {$[(772,780,788),(1089,1100,1111)]$} \\
\hline Ecology & {$[(283,285,287),(639,643,647)]$} & {$[(303,305,307),(995,1002,1009)]$} & {$[(323,325,327),(950,956,962)]$} \\
\hline
\end{tabular}

minimum water supply for ecological sector is satisfied, the total water-allocation amount to ecological user would be $[597,3251] \times 10^{6} \mathrm{~m}^{3}$ over the planning horizon. Such minimum water requirement for ecological sector could alleviate the conflict between ecological conservation and economic development, and develop regional ecological sustainability. In summary, among these water users, agricultural irrigation and ecological requirement would account for a substantial proportion of the total water resources. Several measures regarding water saving and increased water recycling rates would be promoted in the local agricultural production, especially in arid region (i.e., Kaidu-kongque watershed). The obtained solutions suggest that useful information regarding various activities and investments could be provided through the foreseen information that is needed to make decisions.

Figure 5 shows that the system benefits under different $p$-necessity levels through ISFO model under the basic case. The results demonstrate that different $p$-necessity levels result in varied system benefits, which could reflect expected system benefit preference and risk-averse attitude of decision makers. For example, the system benefit would be diminished from $\$[7.51,27.64] \times 10^{9}$ to $\$[7.45,27.51] \times 10^{9}, \$[7.38$, $27.38] \times 10^{9}, \$[7.32,27.25] \times 10^{9}, \$[7.25,27.12] \times 10^{9}, \$$ $[7.18,27.02] \times 10^{9}, \$[7.15,26.93] \times 10^{9}, \$[7.13,26.89] \times 10^{9}$ and $\$[7.12,26.87] \times 10^{9}$, with incremental $p$-necessity levels from 0.70 to $0.75,0.80,0.85,0.90,0.95,0.975,0.99$ and 1 , respectively. If the decision makers prefer to obtain the maximized expected system benefit with high certainty (i.e., necessity), a higher $p$-necessity level would be determined, indicating conservative attitude towards the expected system benefit and decreased uncertainty for the imprecise objective.
On the contrary, the lower $p$-necessity level and increased uncertainty for the imprecise objective would correspond to optimistic attitude on the expected system benefit. At the same time, the uncertain system benefits are presented in term of interval values. The lower bounds of solutions indicate an optimistic estimation of decision makers, while upper bounds show a conservative attitude. In summary, the decision variables with $p$-necessity levels and interval values are beneficial for justifying the decision schemes for associated activities and investments through incorporation of their implicit knowledge, evading the risk of water shortage, environment degradation, and system failure and thereby obtaining maximized system benefits.

4.2. Policy Analysis for Ecological Water Allocation. The study area is threatened by serious water shortages and extremely vulnerable ecological system, while ecological environment plays a significant role in the regional sustainable development. Policy analysis associated with different water resources management based on minimum water requirement for ecological sector is essential to make in-depth analysis. Table 4 presents the solutions of optimized water allocations for ecological sector under various policies and scenarios. The results demonstrate that diverse policies for allocating water resources would yield different water shortages and water-allocation patterns for the ecological sector. For instance, when a minimum amount for ecological water supply is not considered, ecological water supply would first be reduced. The amount of ecological water supply would be zero under Case 2, when stream flows are low and medium in all of the three periods. When the stream flows are high 


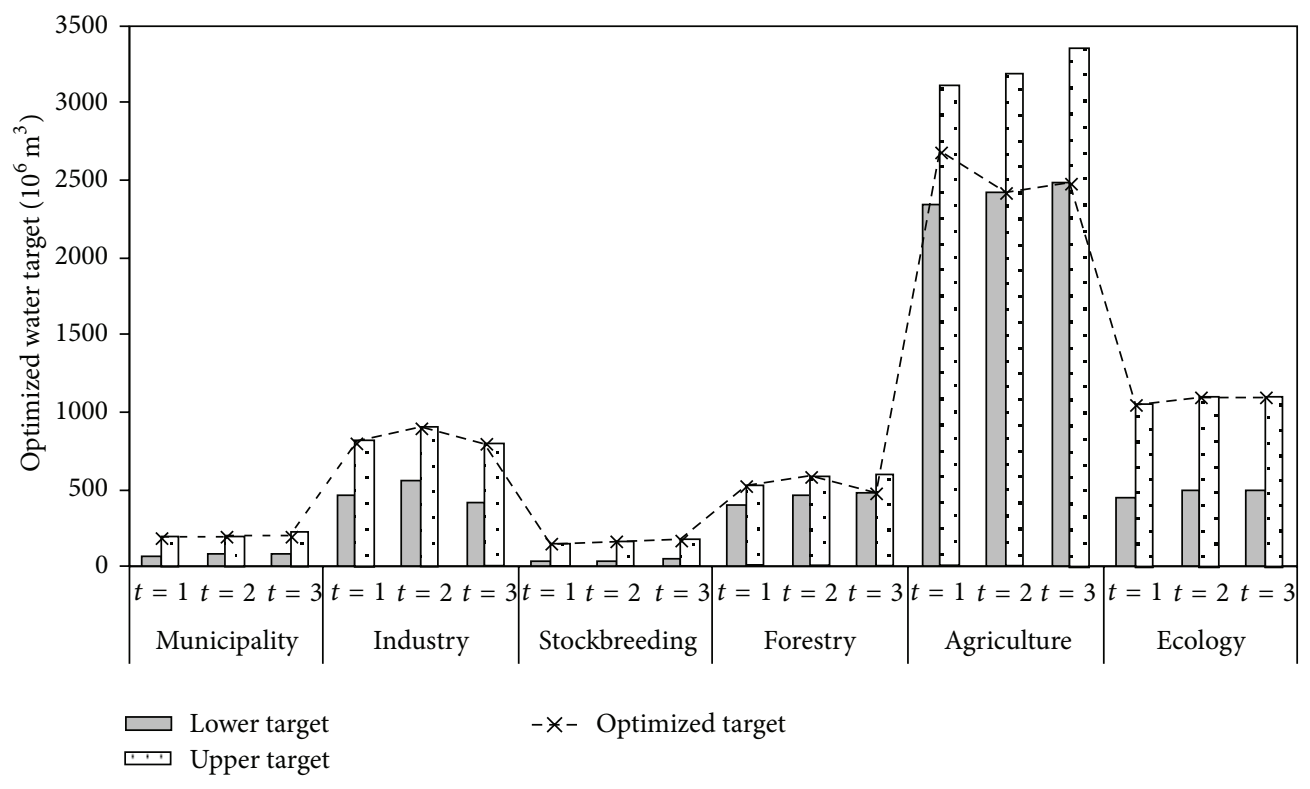

FIGURE 3: Optimized water-allocation targets for different users.

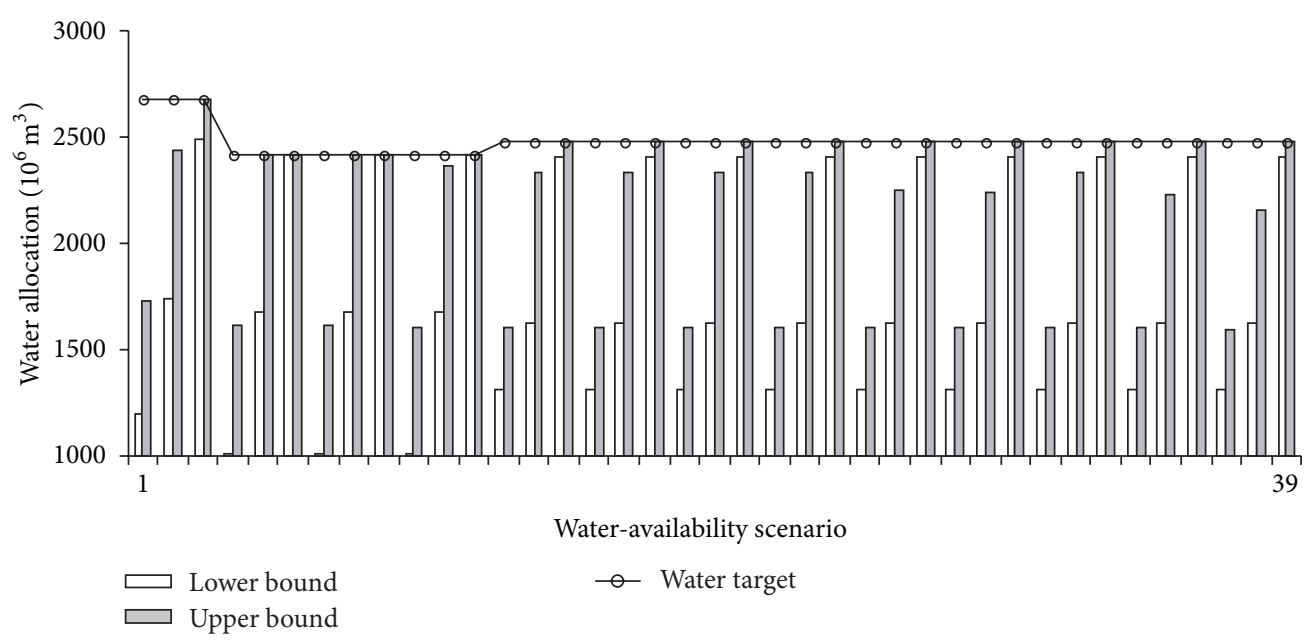

FIGURE 4: Water-allocation plans for agricultural sector.

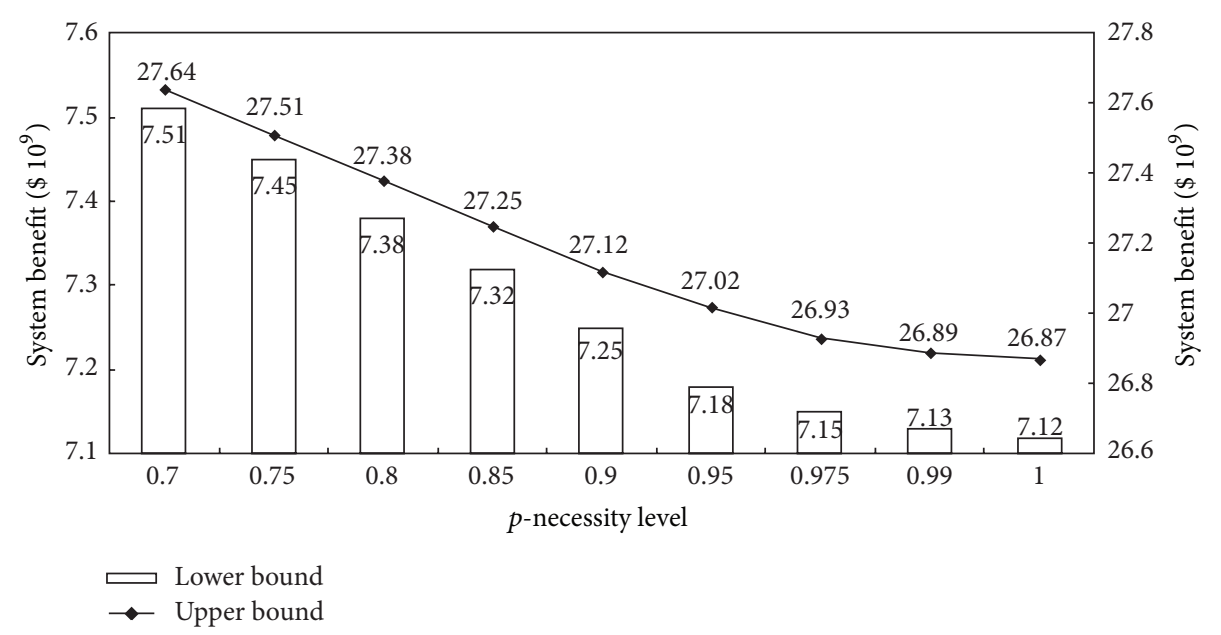

FIGURE 5: System benefits under different $p$-necessity levels. 
TABLE 4: Results of ecological water allocation under different policies.

\begin{tabular}{|c|c|c|c|c|}
\hline \multirow[t]{2}{*}{ Flow level } & \multirow{2}{*}{$\begin{array}{c}\text { Probability } \\
(\%)\end{array}$} & \multirow[t]{2}{*}{ Period } & \multicolumn{2}{|c|}{$\begin{array}{l}\text { Optimized water allocation } \\
\qquad\left(10^{6} \mathrm{~m}^{3}\right)\end{array}$} \\
\hline & & & Case 1 & Case 2 \\
\hline $\bar{L}$ & 30.4 & 1 & 105 & 0 \\
\hline M & 45.5 & 1 & {$[105,113]$} & 0 \\
\hline $\mathrm{H}$ & 24.1 & 1 & {$[105,1051]$} & {$[0,1051]$} \\
\hline LL & 11.24 & 2 & 114 & 0 \\
\hline LM & 15.26 & 2 & 114 & 0 \\
\hline LH & 3.89 & 2 & {$[378,1100]$} & {$[252,1100]$} \\
\hline ML & 16.83 & 2 & 114 & 0 \\
\hline MM & 22.84 & 2 & {$[114,135]$} & 0 \\
\hline $\mathrm{MH}$ & 5.82 & 2 & {$[378,1100]$} & {$[252,1100]$} \\
\hline $\mathrm{HL}$ & 8.91 & 2 & {$[114,120]$} & 0 \\
\hline $\mathrm{HM}$ & 12.10 & 2 & {$[114,205]$} & 0 \\
\hline $\mathrm{HH}$ & 3.08 & 2 & {$[378,1100]$} & {$[252,1100]$} \\
\hline LLL & 1.96 & 3 & 114 & 0 \\
\hline LLM & 5.51 & 3 & 114 & 0 \\
\hline LLH & 3.80 & 3 & {$[114,1065]$} & {$[45,1065]$} \\
\hline LML & 2.66 & 3 & 114 & 0 \\
\hline LMM & 7.48 & 3 & 114 & 0 \\
\hline $\mathrm{LMH}$ & 5.13 & 3 & {$[114,1065]$} & {$[45,1065]$} \\
\hline LHL & 0.68 & 3 & 114.4 & 0 \\
\hline LHM & 1.91 & 3 & 114.4 & 0 \\
\hline LHH & 1.31 & 3 & {$[114,1065]$} & {$[45,1065]$} \\
\hline MLL & 2.93 & 3 & 114 & 0 \\
\hline MLM & 8.25 & 3 & 114 & 0 \\
\hline MLH & 5.66 & 3 & {$[114,1065]$} & {$[45,1065]$} \\
\hline MML & 3.97 & 3 & {$[114,115]$} & 0 \\
\hline MMM & 11.19 & 3 & {$[114,197]$} & 0 \\
\hline $\mathrm{MMH}$ & 7.67 & 3 & {$[114,1065]$} & {$[45,1065]$} \\
\hline MHL & 1.01 & 3 & 114 & 0 \\
\hline MHM & 2.85 & 3 & {$[114,208]$} & 0 \\
\hline $\mathrm{MHH}$ & 1.96 & 3 & {$[114,1082]$} & {$[45,1065]$} \\
\hline HLL & 1.56 & 3 & 114 & 0 \\
\hline HLM & 4.37 & 3 & 114 & 0 \\
\hline HLH & 3.00 & 3 & {$[114,1065]$} & {$[45,1065]$} \\
\hline HML & 2.11 & 3 & {$[114,119]$} & 0 \\
\hline HMM & 5.93 & 3 & {$[114,217]$} & 0 \\
\hline $\mathrm{HMH}$ & 4.06 & 3 & {$[114,1085]$} & {$[45,1065]$} \\
\hline HHL & 0.54 & 3 & {$[114,124]$} & 0 \\
\hline HHM & 1.51 & 3 & {$[114,220]$} & 0 \\
\hline $\mathrm{HHH}$ & 1.04 & 3 & {$[114,1090]$} & {$[45,1065]$} \\
\hline
\end{tabular}

The symbols of $\mathrm{L}, \mathrm{M}$, and $\mathrm{H}$ indicate that the stream flows are low, medium, and high (i.e., LMH denotes that the stream flow are low in period 1, medium in period 2, and high in period 3).

in all of the three periods, the total water-allocation amount to ecological sector under Case 1 and Case 2 would be in the range of $[580,3241] \times 10^{6}$ and $[297,3216] \times 10^{6} \mathrm{~m}^{3}$ over the planning horizon, respectively. In general, under Case 2, the water allocation to ecological sector would first be reduced in case of insufficient water (due to its lowest economic benefit); then, the water shortage would be passed to the agricultural sector. Under Case 1, some water would still be allocated to the ecological sector even under very disadvantageous conditions (i.e., very low inflow). Therefore, water-management policies associated with a minimum ecological water requirement is beneficial for minimizing unfair competition among multiple users and recovering the ecological system in the lower beaches of the river.

At the same time, the solutions of ecological waterallocation policies indicate that owing to benefit and penalty effects on the water-allocation patterns, interactions exist among water supplies for multiple competing users when water deficits occur. The results imply that when a watermanagement policy associated with a minimum ecological water requirement was undertaken, the amount of agricultural water allocation would be decreased, particularly under low and medium stream flow levels. Figure 6 shows the agricultural water allocations under Case 1 and Case 2. For example, when stream flows are medium in all of the three periods, the water allocation to agricultural sector would be $[5053,7110] \times 10^{6} \mathrm{~m}^{3}$ under Case 1 and $[5386,7555] \times 10^{6} \mathrm{~m}^{3}$ under Case 2, respectively. Besides, the agricultural waterallocation target would be in the range of $[7235,9663] \times$ $10^{6} \mathrm{~m}^{3}$ over the planning horizon. This indicates that there would be a marked water shortage for agricultural sector when the minimum ecological water requirement was regulated. Moreover, the water-management policy associated with a minimum ecological water requirement would be helpful for altering traditional agricultural irrigation mode, increasing the water efficiency in the agricultural production and achieving optimal allocation of water resources utilization in arid and semiarid regions. For the municipal, industrial and stockbreeding sectors, the allotment would not be reduced. This is attributed to the fact that these users could bring higher benefits when water demands are satisfied and higher penalties when promised targets are not delivered. The municipal and industrial water play significant roles in living standard and economic development.

In addition, various water-management policies would result in different system benefits (when water was allocated to users). The system benefit under Case 2 would be $\$[7.29$, $27.24] \times 10^{9}$, which is far larger than the Case $1(\$[7.25$, 27.12] $\times 10^{9}$ ). This indicates that ecological sector brings the lowest benefit to the region economic development. Decision makers would usually prefer to allocate the water resources to the users which could bring high benefits. However, the study area is facing increasingly serious water shortages, leading to continued vegetation degradation and human-induced soil erosion. The limited water resources should be allocated in more efficient and sustainable ways. Therefore, willingness to accept a low system benefit (and a low violation risk) could guarantee meeting the ecological water requirement, while a strong desire to obtain a high system benefit could lead to a high risk of ecological water shortage, vegetation loss, and environment degradation.

\section{Conclusions}

In this study, an interval-stochastic fractile optimization (ISFO) model has been proposed for developing optimal water-resources management strategies under multiple 


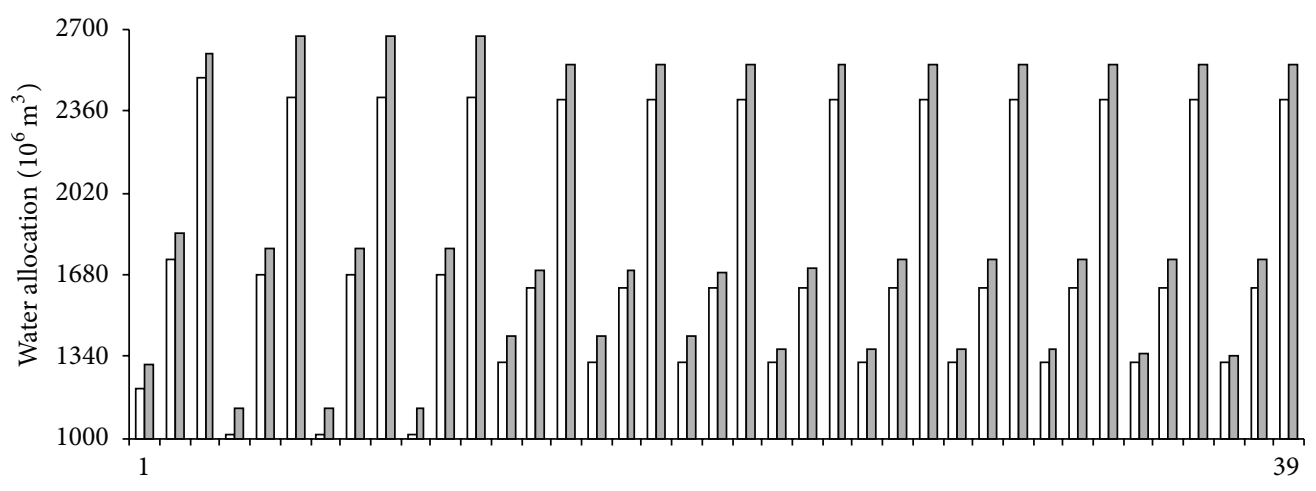

Water-availability scenario

(a) Lower bound

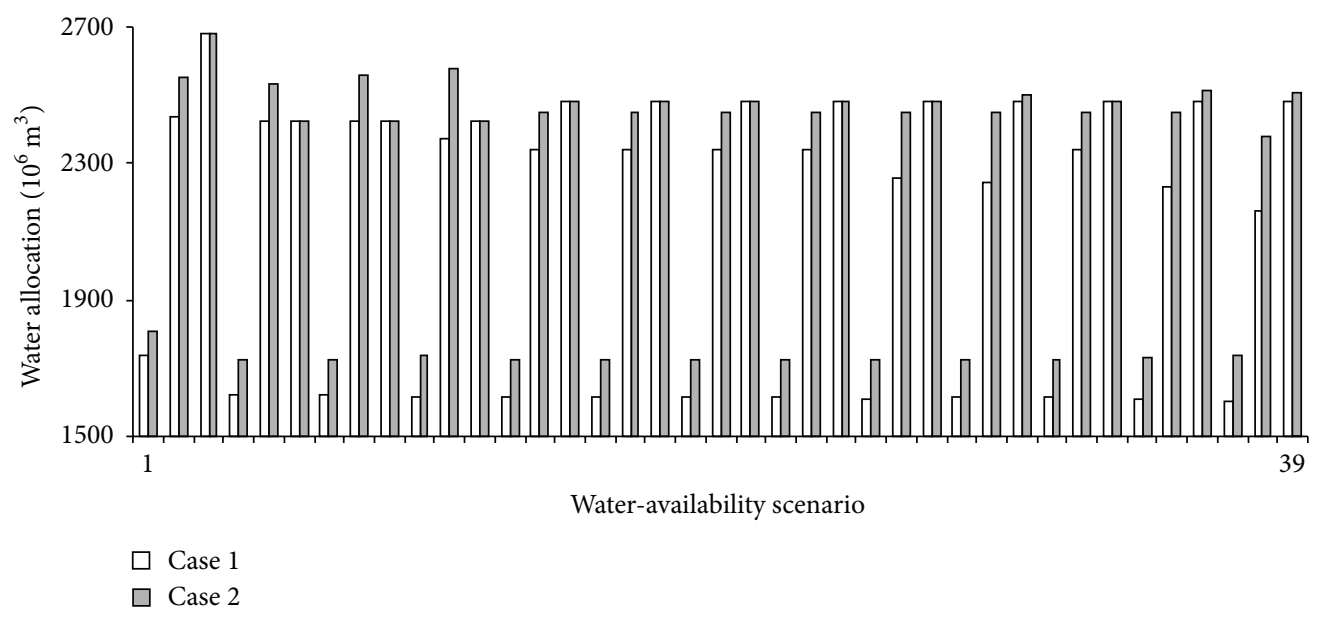

(b) Upper bound

FIgURE 6: Agricultural water allocation under different policies.

uncertainties. The ISFO model can not only handle uncertainties presented in terms of probability distributions and intervals with possibility distribution boundary but also quantify subjective information (i.e., expected system benefit preference and risk-averse attitude) from different decision makers. It can be utilized for analyzing various policy scenarios associated with different levels of economic consequences when the promised targets are violated. The ISFO model is applied to a real case of water-resources systems planning in Kaidu-kongque watershed, China, and a number of scenarios with different ecological water-allocation policies under varied $p$-necessity fractiles are analyzed. Results indicate that different policies for ecological water allocation can lead to varied water supplies, economic penalties, and system benefits. The solutions obtained can help decision makers identify optimized water-allocation alternatives, alleviate the water supply-demand conflict, and achieve socioeconomic and ecological sustainability, particularly when limited water resources are available for multiple competing users.

The study is the first attempt to propose an ISFO model and apply it to water-resources systems planning in Kaidukongque watershed. Nevertheless, there are still several factors that need to be further considered in future research.
Firstly, economic parameters are presented by fuzzy triangular numbers with possibility distribution. More sophisticated solution methods should be proposed to tackle imprecise parameters with nonlinear possibility distribution. Secondly, the dynamic system variations (i.e., nonsequential interactions) are reflected under a multistage context. Advanced methods would be desired to permit revised decisions in each stage based on sequentially realizations for uncertain events. Thirdly, the ISFO model is a single objective model subjected to a set of constraint. Multiobjective programming method should be considered for multicriterion decision analysis with multiple objectives. Therefore, future research needs to be conducted into incorporating powerful optimization techniques within the modeling framework to further enhance the capabilities of the ISFO model.

\section{Acknowledgments}

This research was supported by the National Natural Science Foundation for Distinguished Young Scholar (51225904), the Open Research Fund Program of State Key Laboratory of Hydroscience and Engineering (sklhse-2012-A-03), the National High-Tech R\&D (863) Program (2012AA091103), 
and the Program for Innovative Research Team in University (IRT1127). The authors are grateful to the editor and the anonymous reviewers for their insightful and helpful comments and suggestions.

\section{References}

[1] C. Bao and C. L. Fang, "Water resources constraint force on urbanization in water deficient regions: a case study of the Hexi Corridor, arid area of NW China," Ecological Economics, vol. 62, no. 3-4, pp. 508-517, 2007.

[2] E. Kondili, J. K. Kaldellis, and C. Papapostolou, "A novel systemic approach to water resources optimisation in areas with limited water resources," Desalination, vol. 250, no. 1, pp. 297301, 2010.

[3] Y. Yang, Y. Chen, W. Li, M. Wang, and G. Sun, "Impacts of climatic change on river runoff in Northern Xinjiang of China over last fifty years," Chinese Geographical Science, vol. 20, no. 3, pp. 193-201, 2010.

[4] L. Jing and B. Chen, "Field investigation and hydrological modelling of a subarctic wetland-the Deer River watershed," Journal of Environmental Informatics, vol. 17, no. 1, pp. 36-45, 2011.

[5] L. Y. Su, P. Christensen, and J. L. Liu, "Comparative study of water resource management and policies for ecosystems in China and Denmark," Journal of Environmental Informatics, vol. 21, no. 1, pp. 72-83, 2013.

[6] I. Maqsood, G. H. Huang, and J. S. Yeomans, "An intervalparameter fuzzy two-stage stochastic program for water resources management under uncertainty," European Journal of Operational Research, vol. 167, no. 1, pp. 208-225, 2005.

[7] L. N. Sethi, S. N. Panda, and M. K. Nayak, "Optimal crop planning and water resources allocation in a coastal groundwater basin, Orissa, India," Agricultural Water Management, vol. 83, no. 3, pp. 209-220, 2006.

[8] M. Bravo and I. Gonzalez, "Applying stochastic goal programming: a case study on water use planning," European Journal of Operational Research, vol. 196, no. 3, pp. 1123-1129, 2009.

[9] X. H. Zhang, H. W. Zhang, B. Chen, H. C. Guo, G. Q. Chen, and B. A. Zhao, "An inexact-stochastic dual water supply programming model," Communications in Nonlinear Science and Numerical Simulation, vol. 14, no. 1, pp. 301-309, 2009.

[10] Y. P. Li and G. H. Huang, "Fuzzy-stochastic-based violation analysis method for planning water resources management systems with uncertain information," Information Sciences, vol. 179, no. 24, pp. 4261-4276, 2009.

[11] S. Galelli and R. Soncini-Sessa, "Combining metamodelling and stochastic dynamic programming for the design of reservoir release policies," Environmental Modelling \& Software, vol. 25, no. 2, pp. 209-222, 2010.

[12] Y. P. Li, G. H. Huang, and S. L. Nie, "Optimization of regional economic and environmental systems under fuzzy and random uncertainties," Journal of Environmental Management, vol. 92, no. 8, pp. 2010-2020, 2011.

[13] Y. P. Li, G. H. Huang, S. L. Nie, and X. Chen, "A robust modeling approach for regional water management under multiple uncertainties," Agricultural Water Management, vol. 98, no. 10, pp. 1577-1588, 2011.

[14] A. A. Gaivoronski, G. M. Sechi, and P. Zuddas, "Balancing costrisk in management optimization of water resource systems under uncertainty," Physics and Chemistry of the Earth, Parts A/B/C, vol. 42-44, pp. 98-107, 2012.
[15] D. W. Watkins Jr, D. C. McKinney, L. S. Lasdon, S. S. Nielsen, and Q. W. Martin, "A scenario-based stochastic programming model for water supplies from the highland lakes," International Transactions in Operational Research, vol. 7, pp. 211-230, 2000.

[16] K. W. Harrison, "Two-stage decision-making under uncertainty and stochasticity: Bayesian programming," Advances in Water Resources, vol. 30, no. 3, pp. 641-664, 2007.

[17] Y. P. Li, G. H. Huang, Y. F. Huang, and H. D. Zhou, "A multistage fuzzy-stochastic programming model for supporting sustainable water-resources allocation and management," Environmental Modelling \& Software, vol. 24, no. 7, pp. 786-797, 2009.

[18] Y. Xu, G. H. Huang, and T. Y. Xu, "Inexact management modeling for urban water supply systems," Journal of Environmental Informatics, vol. 20, no. 1, pp. 34-43, 2012.

[19] M. Housh, A. Ostfeld, and U. Shamir, "Limited multi-stage stochastic programming for managing water supply systems," Environmental Modelling \& Software, vol. 41, pp. 53-64, 2013.

[20] M. V. F. Pereira and L. M. V. G. Pinto, "Multi-stage stochastic optimization applied to energy planning," Mathematical Programming, vol. 52, no. 1-3, pp. 359-375, 1991.

[21] S. Ahmed, A. J. King, and G. Parija, "A multi-stage stochastic integer programming approach for capacity expansion under uncertainty," Journal of Global Optimization, vol. 26, no. 1, pp. 3-24, 2003.

[22] J. Dupačová, "Applications of stochastic programming: achievements and questions," European Journal of Operational Research, vol. 140, no. 2, pp. 281-290, 2002.

[23] M. Inuiguchi and J. Ramík, "Possibilistic linear programming: a brief review of fuzzy mathematical programming and a comparison with stochastic programming in portfolio selection problem," Fuzzy Sets and Systems, vol. 111, no. 1, pp. 3-28, 2000.

[24] M. Inuiguchi and T. Tanino, "Portfolio selection under independent possibilistic information," Fuzzy Sets and Systems, vol. 115, no. 1, pp. 83-92, 2000.

[25] N. Arbaiy and J. Watada, "Fractile optimization approach for possibilistic programming problem," in Proceedings of the International Conference on Man-Machine Systems (ICoMMS '12), 2012.

[26] M. Inuiguchi, "Robust optimization by fuzzy linear programming," in Managing Safety of Heterogeneous Systems, vol. 658 of Lecture Notes in Economics and Mathematical Systems, pp. 219239, Springer, 2012.

[27] M. Inuiguchi, "Fuzzy programming approaches to robust optimization," in Proceedings of the 9th international conference on Modeling Decisions for Artificial Intelligence (MDAI '12), pp. 1112, 2012.

[28] H. Katagiri, M. Sakawa, K. Kato, and S. Ohsaki, "An interactive fuzzy satisficing method based on the fractile optimization model using possibility and necessity measures for a fuzzy random multiobjective linear programming problem," Electronics and Communications in Japan, Part III, vol. 88, no. 5, pp. 20-28, 2005.

[29] X. Zhang, G. H. Huang, and X. Nie, "Robust stochastic fuzzy possibilistic programming for environmental decision making under uncertainty," Science of the Total Environment, vol. 408, no. 2, pp. 192-201, 2009.

[30] G. H. Huang and M. F. Cao, "Analysis of solution methods for interval linear programming," Journal of Environmental Informatics, vol. 17, no. 2, pp. 54-64, 2011.

[31] Y. R. Fan and G. H. Huang, "A robust two-step method for solving interval linear programming problems within an 
environmental management context," Journal of Environmental Informatics, vol. 19, no. 1, pp. 1-9, 2012.

[32] Y. P. Li, G. H. Huang, and S. L. Nie, "Planning water resources management systems using a fuzzy-boundary intervalstochastic programming method," Advances in Water Resources, vol. 33, no. 9, pp. 1105-1117, 2010.

[33] Y. Zhou, Y. P. Li, G. H. Huang, and Y. Zhou, "A robust approach for planning electric power systems associated with environmental policy analysis," Electric Power Systems Research, vol. 95, pp. 99-111, 2013.

[34] D. Dubois and H. Prade, Possibility Theory: An Approach to Computerized Processing of Uncertainty, Plenum Press, New York, NY, USA, 1988.

[35] L. A. Zadeh, "Fuzzy sets as a basis for a theory of possibility," Fuzzy Sets and Systems, vol. 1, no. 1, pp. 3-28, 1978.

[36] H. Tanaka, P. Guo, and H. J. Zimmermann, "Possibility distributions of fuzzy decision variables obtained from possibilistic linear programming problems," Fuzzy Sets and Systems, vol. 113, no. 2, pp. 323-332, 2000.

[37] S. A. Torabi and E. Hassini, "An interactive possibilistic programming approach for multiple objective supply chain master planning," Fuzzy Sets and Systems, vol. 159, no. 2, pp. 193-214, 2008.

[38] Z. F. Liu, G. H. Huang, and N. Li, "A dynamic optimization approach for power generation planning under uncertainty," Energy Sources, Part A, vol. 30, no. 14-15, pp. 1413-1431, 2008.

[39] S. A. Torabi and E. Hassini, "An interactive possibilistic programming approach for multiple objective supply chain master planning," Fuzzy Sets and Systems, vol. 159, no. 2, pp. 193-214, 2008.

[40] H. L. Xu, M. Ye, and J. M. Li, "Changes in groundwater levels and the response of natural vegetation to transfer of water to the lower reaches of the Tarim River," Journal of Environmental Sciences, vol. 19, no. 10, pp. 1199-1207, 2007.

[41] Y. Huang, Y. P. Li, X. Chen, A. M. Bao, and Y. G. Ma, "A multistage simulation-based optimization model for water resources management in Tarim River Basin, China," Stochastic Environmental Research and Risk Assessment, vol. 27, no. 1, pp. 147-158, 2013.

[42] Y. P. Li, G. H. Huang, Z. F. Yang, and S. L. Nie, "IFMP: interval-fuzzy multistage programming for water resources management under uncertainty," Resources, Conservation and Recycling, vol. 52, no. 5, pp. 800-812, 2008.

[43] Y. P. Li, G. H. Huang, X. H. Nie, and S. L. Nie, "An inexact fuzzyrobust two-stage programming model for managing sulfur dioxide abatement under uncertainty," Environmental Modeling and Assessment, vol. 13, no. 1, pp. 77-91, 2008.

[44] Statistical Bureau of Bayangol Mongol Autonomous Prefecture, Statistical Yearbook of Bayangol, China Statistic Press, Beijing, China, 2000-2009.

[45] Y. P. Li, G. H. Huang, G. Q. Wang, and Y. F. Huang, "FSWM: a hybrid fuzzy-stochastic water-management model for agricultural sustainability under uncertainty," Agricultural Water Management, vol. 96, no. 12, pp. 1807-1818, 2009. 


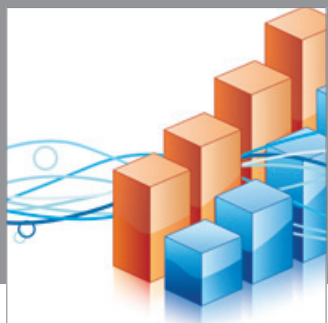

Advances in

Operations Research

mansans

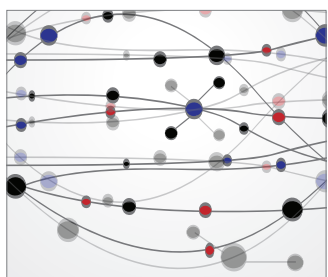

The Scientific World Journal
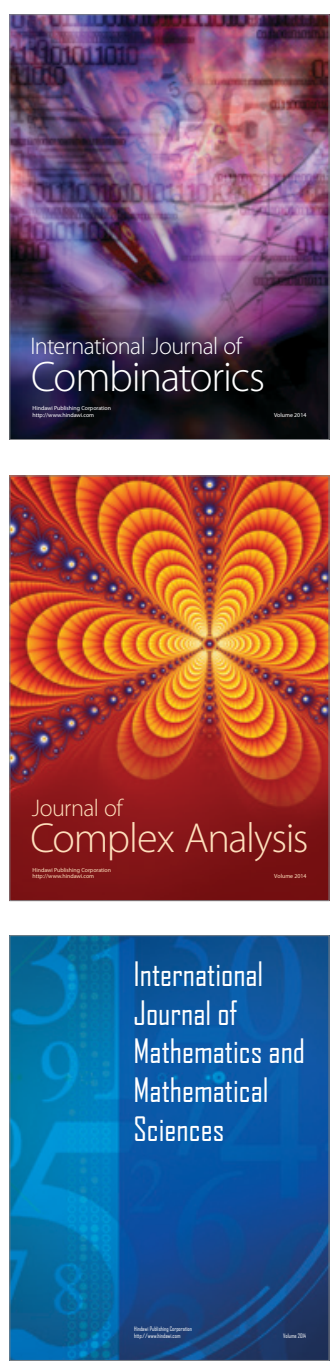
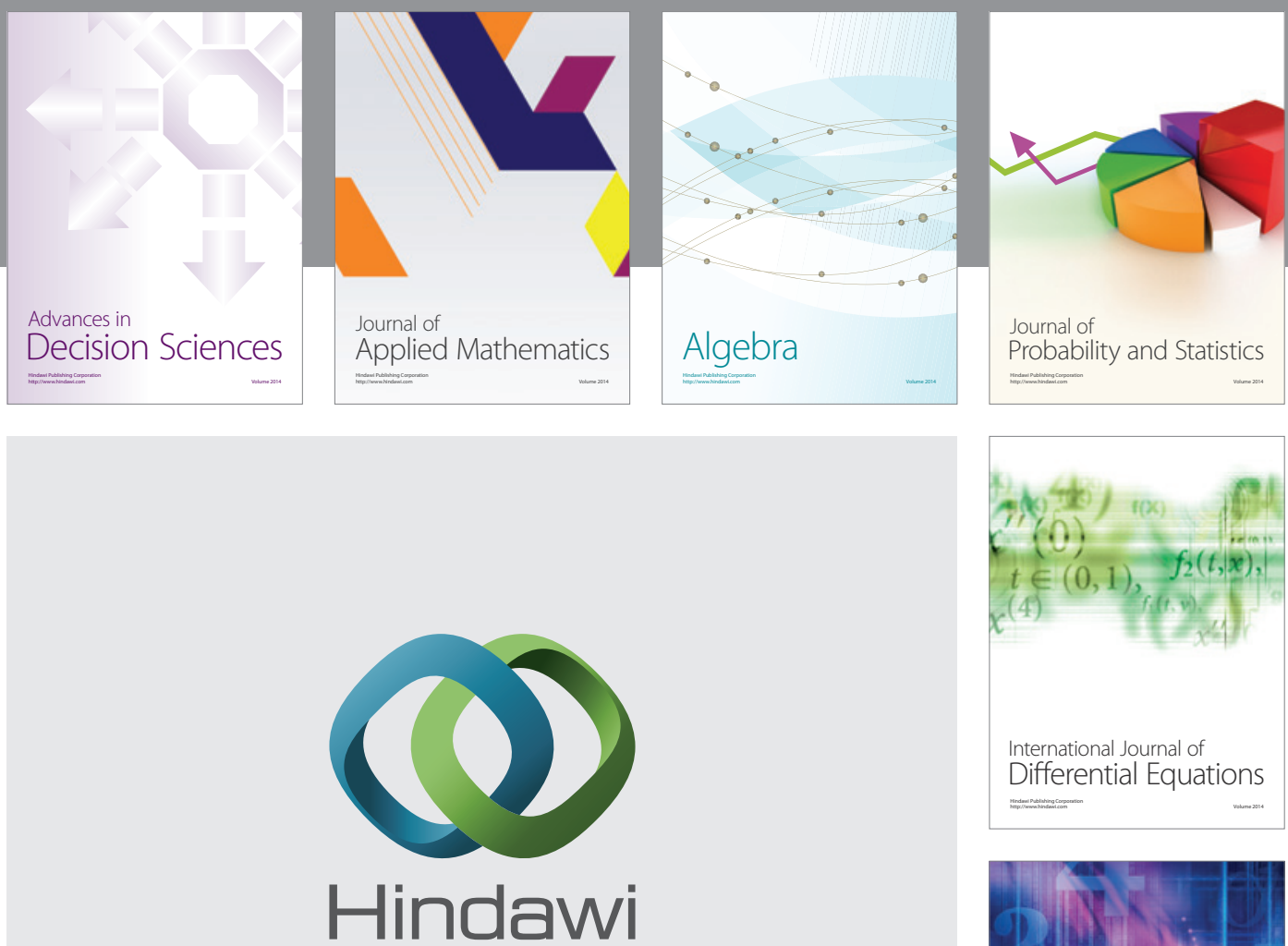

Submit your manuscripts at http://www.hindawi.com
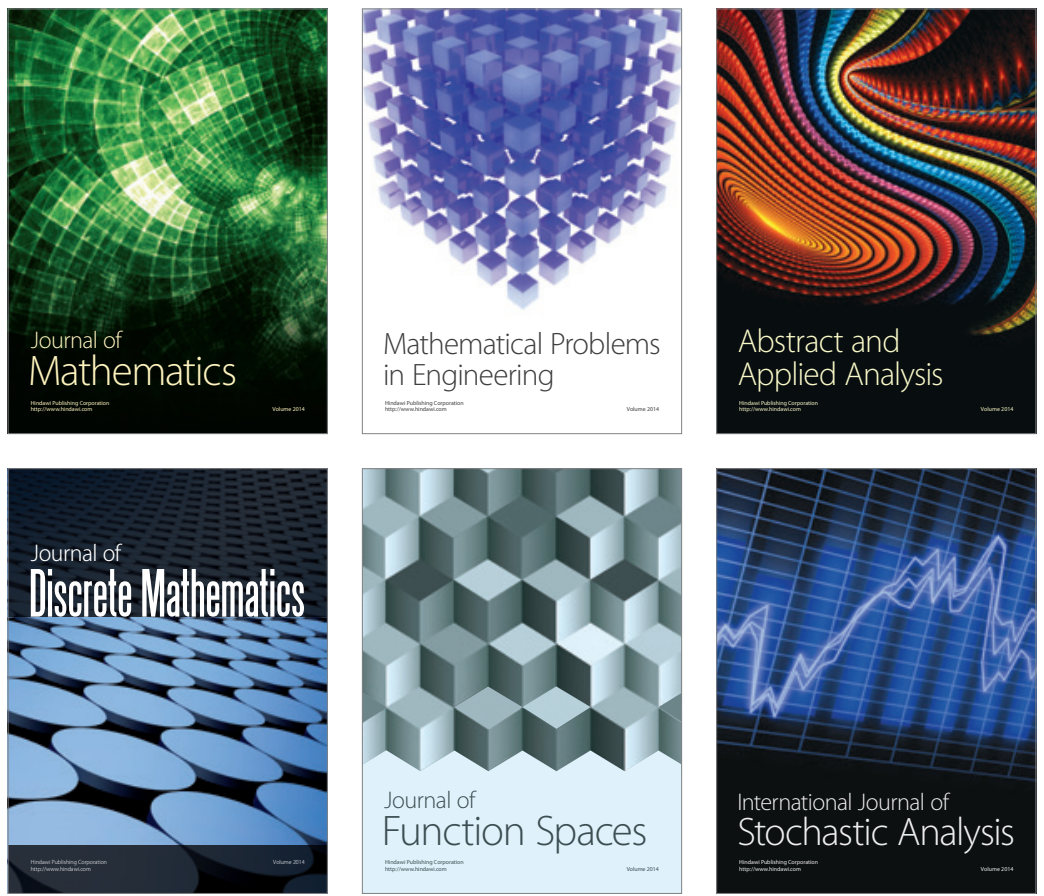

Journal of

Function Spaces

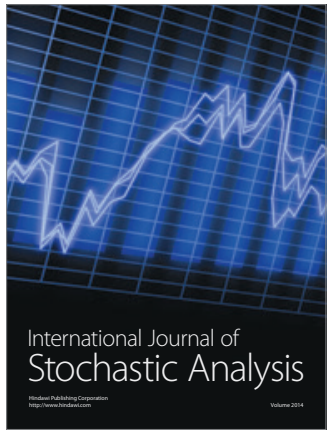

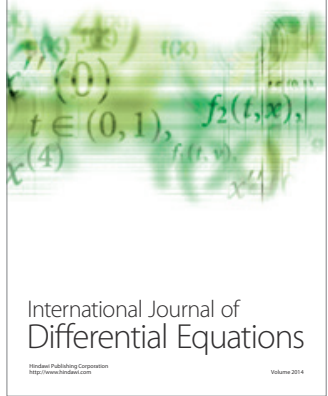
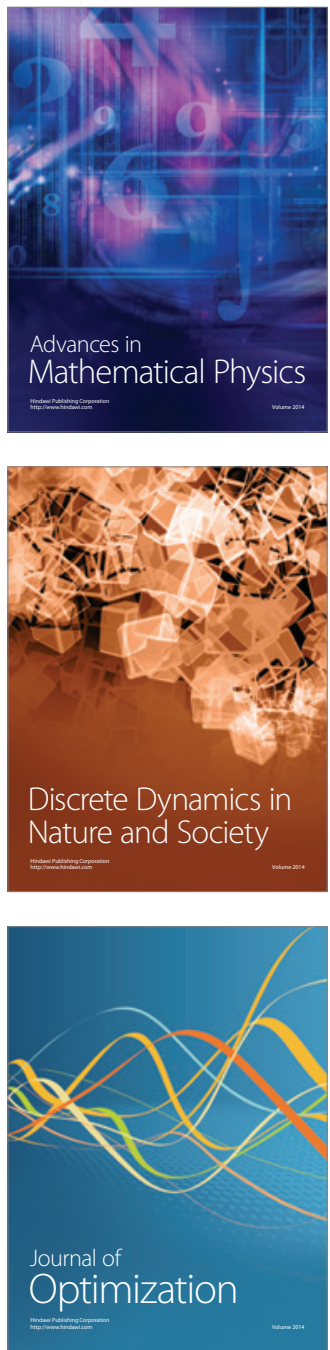